\title{
Validation of commercially available ELISAs for the detection of circulating sclerostin in hemodialysis patients
}

\author{
Sebastian F. Mause ${ }^{* 1}$, Annika Deck ${ }^{1}$, Mark Hennies ${ }^{2}$, Nadine Kaesler $^{3}$, Pieter Evenepoel ${ }^{4}$, \\ William A. Boisvert ${ }^{5}$, Ulf Janssen ${ }^{\#, 6}$, Vincent M. Brandenburg ${ }^{\#, 1}$ \\ ${ }^{1}$ Department of Cardiology, University Hospital of the RWTH Aachen, Germany \\ ${ }^{2}$ TECOmedical AG, Sissach, Switzerland \\ ${ }^{3}$ Department of Nephrology, University Hospital of the RWTH Aachen, Aachen, Germany \\ ${ }^{4}$ Department of Medicine, University Hospital Leuven, Leuven, Belgium \\ ${ }^{5}$ Center for Cardiovascular Research, John A. Burns School of Medicine, University of Hawaii, Honolulu, \\ USA \\ ${ }^{6}$ Department of Nephrology, Klinikum Maria-Hilf, Mönchengladbach, Germany \\ \# These authors contributed equally to this work \\ * Corresponding author: Sebastian F. Mause, MD, Department of Cardiology, Pauwelsstraße 30, D - 52074 \\ Aachen, University Hospital RWTH Aachen, Germany; Phone: +49 (0)241/80 35223; Fax: +49 (0)241/80 \\ 82446; Email: smause@ukaachen.de
}

Submitted: March 25, 2016; Revised: March 30, 2016; Accepted: March 31, 2016; Published: March 31, 2016; Citation: Mause SF, Deck A, Hennies M, Kaesler N, Evenepoel P, Janssen U, Brandenburg VM. Validation of commercially available ELISAs for the detection of circulating sclerostin in hemodialysis patients. Discoveries 2016, January-March; 4(1): e55. DOI: 10.15190/d.2016.2

\begin{abstract}
BACKGROUND: Sclerostin is an endocrine regulator in chronic kidney disease - mineral and bone disorder (CKD-MBD). Validation of assay comparability and pre-analytical handling is mandatory for establishment of sclerostin as a biomarker.
\end{abstract}

METHODS: Blood samples (serum, EDTA, heparin and citrate plasma) were obtained from 12 hemodialysis (HD) patients after the long dialysis interval. Passing-Bablok regression analysis and Bland-Altman difference plots were used to evaluate the agreement between sclerostin levels measured with two commercially available ELISAs from TECOmedical and Biomedica.

RESULTS: Independent of the sample type, the agreement of the two assays was poor with a strong proportional but no systematic bias. Compared to the TECOmedical assay, the Biomedica test yielded almost 2-fold higher sclerostin values throughout all sample types. Spike recovery and linear dilution studies revealed a higher accuracy of the TECOmedical assay (97\% and 96\%) compared tothe Biomedica assay (118\% and 78\%). Sclerostin levels were stable within 4 hours after sample collection, in particular when analyzed in plasma. In contrast to the Biomedica assay, the TECOmedical showed a systematic but no proportional bias between serum and plasma samples with higher values for plasma samples. Among the 3 different plasma samples no systematic error could be documented.

CONCLUSION: Careful consideration of the preanalytical handling and comparative assay validation are necessary to facilitate a more differentiated interpretation of studies reporting circulating sclerostin levels. The presence of a proportional bias demonstrates that in HD patients the two ELISAs for measuring sclerostin should not be used interchangeably. Furthermore, caution is necessary when comparing sclerostin results obtained from different blood sample types. 
Keywords: biomarker, CKD-MBD, haemodialysis, sclerostin, vascular calcification

\section{Abbreviations: \\ Chronic kidney disease - mineral and bone disorder (CKD-MBD), end-stage-renal disease (ESRD) \\ INTRODUCTION}

The close relationship between bone disease, mineral disturbances and cardiovascular disorders in patients with chronic kidney disease (CKD) is increasingly acknowledged and has led to the introduction of the term chronic kidney disease mineral and bone disorder (CKD-MBD) ${ }^{1}$. One of the recently identified regulators involved in CKDMBD is the glycoprotein sclerostin, a soluble Wnt inhibitor produced mainly in osteocytes. Sclerostin inhibits differentiation and function of osteoblasts and possibly interferes with biological signaling that operates in the vessel wall ${ }^{2}$. Sclerostin represents a promising biomarker in CKD-MBD since the physiology of sclerostin is altered during renal insufficiency and since the modulation of the Wnt signaling pathway via sclerostin is involved in the development of renal osteodystrophy and in cardiovascular diseases associated with $\mathrm{CKD}^{3,4}$. Moreover, measurement of circulating sclerostin in patients with end-stage-renal disease (ESRD) revealed associations with bone mineral density ${ }^{5}$, cardiovascular mortality in prospective observational cohort studies ${ }^{6,7}$, and with vascular calcification $^{8-10}$. Of note, some of these studies have reported contradictory results, thus awaiting further confirmation.

In terms of establishing novel biomarkers, assay validation and standardization are essential ${ }^{11}$. Significant disagreement between different assays measuring the same parameter may impair the comparability of results in a clinically meaningful manner. Indeed, a relevant variability among different intact parathyroid hormone assays in patients with ESRD has been confirmed ${ }^{12}$. Previous studies designed to compare two commercially available sclerostin ELISAs, namely the Biomedica and the TECOmedical ELISA, identified in various clinical settings a relevant variation of measured sclerostin values, therefore limiting the clinical interpretation of reported values and compromising the comparability of studies using different immunoassays for sclerostin detection ${ }^{13-16}$. Several additional factors such as pre-analytical handling including the choice of blood sample type, storage time, and matrix interference may affect the quantification of biomarkers. For the robust introduction of sclerostin as a biomarker in CKDMBD it is thus mandatory to further validate the applied assays and to assess the relevance of preanalytical variables particularly in (haemodialysis) HD patients.

\section{MATERIALS AND METHODS}

Sample collection. Human blood samples were obtained with the written, informed consent of the patients and the permission from local ethical committees (RWTH Aachen EC vote number EK 300/11). Blood was drawn by venipuncture after an overnight fast at the end of the long dialysis interval from 12 clinically stable patients with ESRD before undergoing routine hemodialysis. We collected serum and three types of plasma (EDTA, lithium heparin and citrate plasma) in standard monovettes (Sarstedt, Nümbrecht, Germany). Patients (5 men, 7 women) were aged $66.6 \pm 9.1$ years and were on dialysis for a median time of 54 months. For further details regarding the patient population please refer to Supplemental Table 1. Samples were centrifuged after 20, 60, 120 and 240 minutes at room temperature for 10 minutes at $2000 \mathrm{~g}$ and subsequently stored as aliquots at $-80^{\circ} \mathrm{C}$ until assayed. No sample was thawed more than twice and all samples were assayed using the same lot of the respective ELISA Kit.

Sclerostin measurement. Sclerostin levels were measured by two commercially available ELISAs according to the respective manufacturer's instructions. All plates and reagents were from the same lot. Samples were tested in duplicate and the calculated means were used for further statistical analysis. Both the Biomedica assay (Biomedica, Wien, Austria) and the TECOmedical assay (TECOmedical, Sissach, Switzerland) represent two-site immune-capture ELISAs. The characteristics of the assays are summarized in Supplemental Table 2. Of note, values from all patient samples were in the proper range of the respective test. The reported intra-assay and interassay precision of the sclerostin ELISAs are $\leq 7 \%$, respectively $\leq 10 \%$ for the Biomedica assay and $\leq 6 \%$, respectively $\leq 9 \%$ for the TECOmedical assay. 
Stability. Stability is the capability of a sample material to retain the initial property of a measured constituent for a period of time within specified
Statistics. Levels of sclerostin and other parameters are expressed as mean \pm standard deviation (SD). The nonparametric regression procedure of Passing-

Table 1. Stability of various sample types stored for up to $240 \mathrm{~min}$. Results are presented as \% of the baseline (20 min) control \pm SD.

\begin{tabular}{ccccccc|}
$\begin{array}{c}\text { time } \\
(\mathbf{m i n})\end{array}$ & Biomedica & Biomedica & Biomedica & TECOmedical & TECOmedical & TECOmedical \\
& serum & heparin & EDTA & serum & heparin & EDTA \\
$\mathbf{2 0}$ & 100 & 100 & 100 & 100 & 100 & 100 \\
$\mathbf{6 0}$ & $94.6 \pm 12.8$ & $101.8 \pm 3.1$ & $101.0 \pm 1.9$ & $94.8 \pm 14.1$ & $105.2 \pm 2.5$ & $103.6 \pm 6.6$ \\
$\mathbf{1 2 0}$ & $91.2 \pm 10.9$ & $100.6 \pm 6.1$ & $99.0 \pm 3.4$ & $87.8 \pm 9.4$ & $105.0 \pm 5.1$ & $102.4 \pm 6.0$ \\
$\mathbf{2 4 0}$ & $96.6 \pm 19.0$ & $97.4 \pm 3.5$ & $104.2 \pm 10.5$ & $97.6 \pm 22.1$ & $98.4 \pm 13.8$ & $103 \pm 7.3$ \\
\hline
\end{tabular}

limits when the sample is stored under defined conditions. To evaluate the stability of sclerostin in serum as well as in all 3 plasma samples at room temperature, collected blood from 4 patients was left unprocessed for various periods ranging from 20 to $240 \mathrm{~min}$ and subsequently centrifuged and frozen at $-80{ }^{\circ} \mathrm{C}$. Samples were thawed at room temperature and all samples were assayed at the same time.

Accuracy and assessment of matrix effects. The accuracy of highly sensitive biomarker methods is frequently confounded by the presence of endogenous factors in samples causing matrix effects. For determination of the accuracy of the two ELISAs and assessment of matrix effects, we performed linearity analysis as well as spike recovery experiments. Linearity of dilution, also known as parallelism, was evaluated by serially diluting samples with sample diluent (within the range of the standard curve) and comparing observed values with expected values. Samples were diluted to $1: 2$ and 1:4 according to the manufacturer's protocol. Linearity (\%) was assessed as linearity $=100 \mathrm{x}$ observed value / (previous value/dilution factor). Spike recovery is used to determine if the assay is affected by the difference between the diluent used to prepare the standard curve and the biological sample matrix. Analysis of such recovery was performed by spiking samples with a known quantity of purified sclerostin and comparing observed values with expected values. Recovery (\%) was determined as recovery $=100 \mathrm{x}$ (observed value - unspiked sample value) / expected value.
Bablok and concordance correlation coefficient (CCC) were used to evaluate the comparability and the agreement between sclerostin results obtained with the two ELISAs and the different sample types. To demonstrate and visualize the relationship between the differences and the magnitude of measurements we conducted a Bland-Altman analysis ${ }^{17}$. Statistical analysis was performed using the open-source software $\mathrm{R}$ with the mcr package (R Foundation for Statistical Computing, Vienna, Austria) and GraphPad Prism 6 (GraphPad Software, Inc., CA, USA).

\section{RESULTS}

Stability of sclerostin in serum and plasma: influence of pre-analytical storage time. We tested the effect of sample storage at room temperature on the detection of sclerostin in serum and plasma. As shown in Table 1, sclerostin was stable within 4 hours after sample collection when stored as plasma (EDTA, heparin and citrate) with a maximum deviation of $5.2 \%$ (TECOmedical, heparin plasma) and $4.2 \%$ (Biomedica, EDTA plasma). These results indicate that it is feasible to store plasma samples for up to 4 hours before centrifugation and subsequent analysis. When analyzing serum samples, we observed inferior stability compared to plasma with a maximum deviation of $12.2 \%$ (TECOmedical) and 8.8\% (Biomedica).

Dilution and spike recovery. Linearity analysis (linearity-of-dilution) was performed by diluting samples with specimen diluent and comparing observed values with expected values. To further 
Table 2. Dilution linearity (up) and spike recovery (below) expressed as $\%$ of recovery \pm SD.

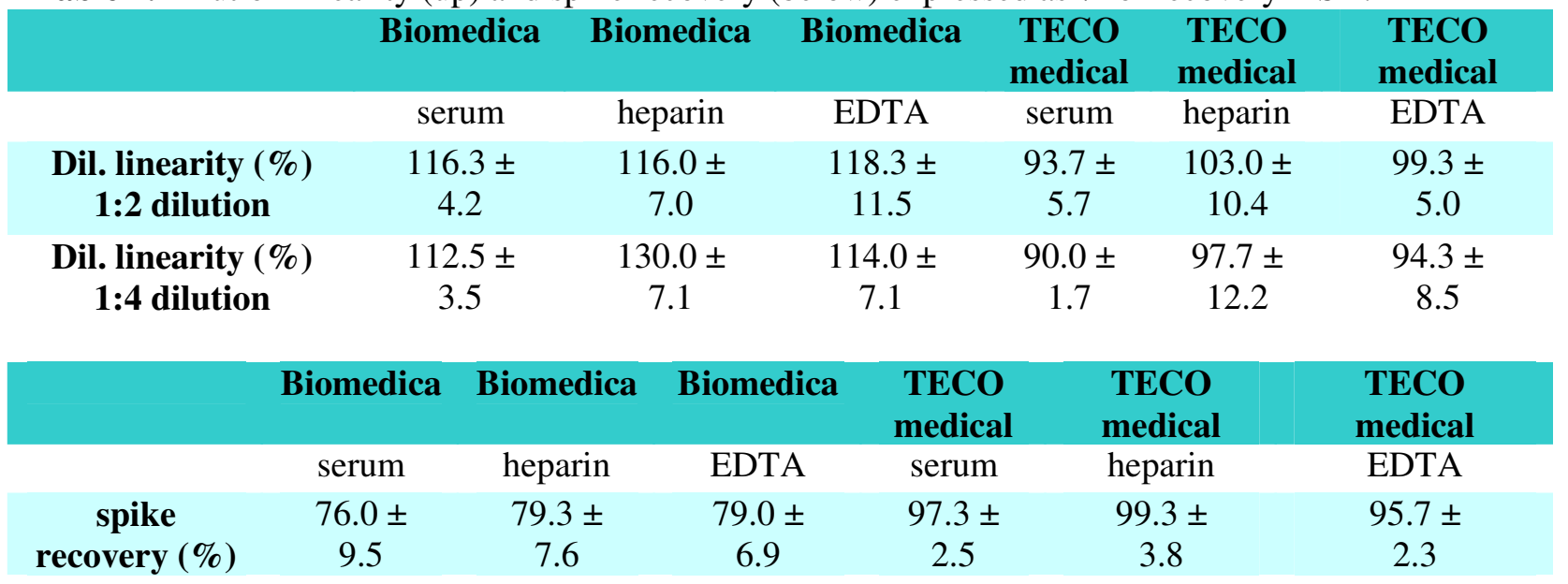

validate the accuracy of the ELISA, spike recovery studies were performed by spiking samples with a known quantity of purified recombinant sclerostin and comparing measured with expected values. Whereas the TECOmedical assay was characterized by a high linearity (dilution recovery of all samples $98.7 \pm 7.0 \%$ for $1: 2$ dilution, $94.0 \pm 7.5 \%$ for $1: 4$ dilution) and a spike recovery in the proper range $(97 \pm 2,9 \%)$, the Biomedica assay showed a significant inferior accuracy with an over-recovery

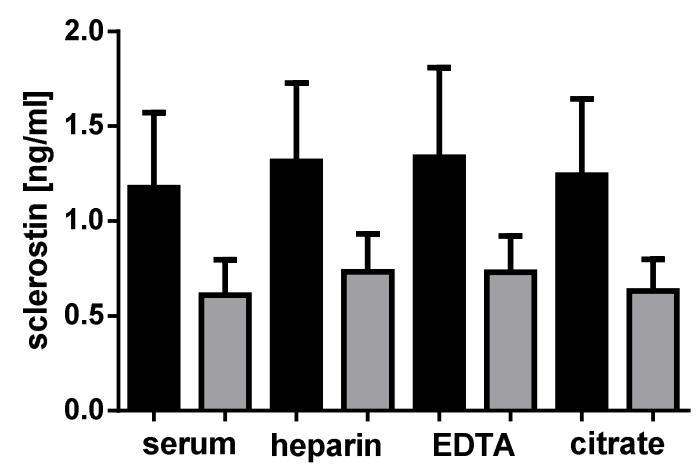

Figure 1. Sclerostin levels as determined by the Biomedica (black bars) and TECOmedical (grey bars) ELISAs in various sample types as indicated. Data represent mean $\pm \mathrm{SD}$

in the dilution series (dilution recovery $116.9 \pm$ $7.6 \%$ for $1: 2$ dilution and $118.8 \pm 5.9 \%$ for $1: 4$ dilution) and an under-recovery in the spike testing (spike recovery $78 \pm 8.1 \%$ ), suggesting a significant matrix interference (Table 2, Supplemental Figure 1 and 2). Of note, all values (spiked and unspiked) were above the lower limit of quantification and on the linear portion of the curve.

Levels of sclerostin. Comparing the two ELISAs, we observed that the Biomedica assay yielded significant higher sclerostin values both in serum and plasma samples (Table 3 and Figure 1). Whereas EDTA plasma sclerostin concentrations measured with the TECOmedical assay were $0.73 \pm$ $0.30 \mathrm{ng} / \mathrm{ml}$ (mean $\pm \mathrm{SD}$ ), EDTA plasma sclerostin levels were $1.34 \pm 0.74 \mathrm{ng} / \mathrm{ml}$ with the Biomedica assay. The difference was most pronounced when determining sclerostin levels in citrate plasma (1.97 fold higher with the Biomedica assay) and least pronounced in heparin plasma (1.79 fold higher with the Biomedica assay). In general, determination of sclerostin concentration in serum yielded the lowest values followed by citrate plasma.

The Bland-Altman plots (Figure 2) revealed that the differences between the two assays enlarge as the level of sclerostin increases, suggesting that the putative overestimation of absolute sclerostin levels using the Biomedica test increases with higher concentrations. In addition, the scatter around the bias line became larger at higher sclerostin levels, indicating an inconsistent variability. The mean difference(=bias)and the respective $95 \%$ confidence intervals $(\mathrm{CI})$ are summarized in Table 4.

In order to further evaluate the comparability and the agreement between sclerostin results obtained with the TECOmedical and Biomedica assay and to elucidate the influence of the chosen 
Table 3. Descriptive statistics of sclerostin measurement stratified by blood sample type and assay

\begin{tabular}{|c|c|c|c|c|c|c|c|c|c|}
\hline & $\begin{array}{c}\text { sample } \\
\text { type }\end{array}$ & $\min$ & $\begin{array}{c}\text { 1st } \\
\text { quart. }\end{array}$ & median & mean & $\begin{array}{c}\text { 3rd } \\
\text { quart }\end{array}$ & $\max$ & SD & n \\
\hline TECOmedical & serum & 0.18 & 0.43 & 0.56 & 0.61 & 0.77 & 1.21 & 0.30 & 12 \\
\hline Biomedica & serum & 0.54 & 0.74 & 0.9 & 1.18 & 1.48 & 2.42 & 0.62 & 12 \\
\hline TECOmedical & heparin & 0.23 & 0.54 & 0.7 & 0.73 & 0.83 & 1.44 & 0.31 & 12 \\
\hline Biomedica & heparin & 0.6 & 0.91 & 1.08 & 1.32 & 1.54 & 2.71 & 0.65 & 12 \\
\hline TECOmedical & EDTA & 0.23 & 0.61 & 0.71 & 0.73 & 0.84 & 1.33 & 0.30 & 12 \\
\hline Biomedica & EDTA & 0.53 & 0.9 & 1.06 & 1.34 & 1.46 & 2.82 & 0.74 & 12 \\
\hline TECOmedical & citrate & 0.21 & 0.5 & 0.61 & 0.63 & 0.73 & 1.14 & 0.26 & 12 \\
\hline Biomedica & citrate & 0.56 & 0.84 & 1.03 & 1.24 & 1.4 & 2.75 & 0.63 & 12 \\
\hline
\end{tabular}

Table 4. Bland-Altman analysis testing the agreement of the Biomedica and TECOmedical ELISAs. $\mathrm{LOA}=$ limit of agreement.

\begin{tabular}{lccccc}
$\begin{array}{l}\text { Biomedica vs. } \\
\text { TECOmedical }\end{array}$ & serum & heparin & EDTA & citrate & all samples \\
bias (mean difference) & 0.568 & 0.583 & 0.607 & 0.610 & 0.592 \\
SD of bias & 0.382 & 0.404 & 0.489 & 0.422 & 0.413 \\
\hline lower LOA (95\% CI) & -0.181 & -0.209 & -0.351 & -0.218 & -0.217 \\
\hline upper LOA (95\% CI) & 1.316 & 1.375 & 1.565 & 1.438 & 1.401
\end{tabular}

sample type, we applied the nonparametric regression procedure of Passing-Bablok.

Independent of the sample type, the agreement of the two assays was poor with a strong proportional but not systematic bias (Table 5, Figure 3). Among all tested sample types, the 95\% $\mathrm{CI}$ interval for the intercept did include 0 , indicating the absence of a systematic error/bias between the two ELISAs, whereas with the exception of heparin plasma (lower CI 0.962) the 95\% confidence interval for the slope did not include 1 , revealing the presence of a proportional error between the Biomedica and TECOmedical assay. The proportional bias was largest in EDTA plasma (slope 2.50) and smallest in heparin plasma samples (slope 1.90). The resulting equation of PassingBablok analysis for the combination of all sclerostin measurements independent of the sample type was $\mathrm{y}=0.064+2.024 \mathrm{x}(95 \% \mathrm{CI}$ of intercept -0.460 and $0.142 ; 95 \%$ CI of slope 1.571 and 2.633). Concordance correlation coefficient for all sample types was 0.39 , the Pearson correlation coefficient 0.885 . Passing-Bablok analysis identified neither a systematic nor a proportional difference between the four blood types when using the Biomedica assay (Table 6 and Figure 4A,B). When comparing each of the three plasma types with serum, in all cases the $95 \%$ confidence interval for the intercept did include 0 and the slope did include 1, demonstrating a good agreement among the various sample types. The same could be documented when directly comparing the 3 different plasma types with each other. In contrast, analysis of the TECOmedical assay provided evidence for a systematic but not proportional bias between serum and each of the plasma samples, with generally higher values for plasma probes (Table 6 and Figure 4A,B). Among the three different plasma samples no systematic error could be observed. Between citrate vs. heparin and citrate vs. EDTA, but not between heparin vs. EDTA the 95\% confidence interval for the slope did not include 1 , indicating a proportional error with lower sclerostin results obtained in citrate plasma samples compared to EDTA and heparin plasma samples.

\section{DISCUSSION}

As sclerostin represents a promising novel biomarker for the multifaceted entity of CKD$\mathrm{MBD}^{3}$, reliable measurement of circulating sclerostin is of increasing interest. However, various sclerostin immunoassays were found to yield inconsistent results ${ }^{16,18}$. 
serum

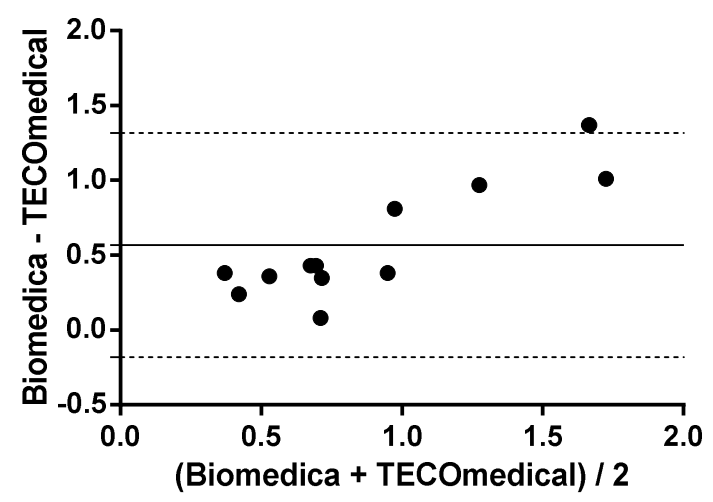

EDTA

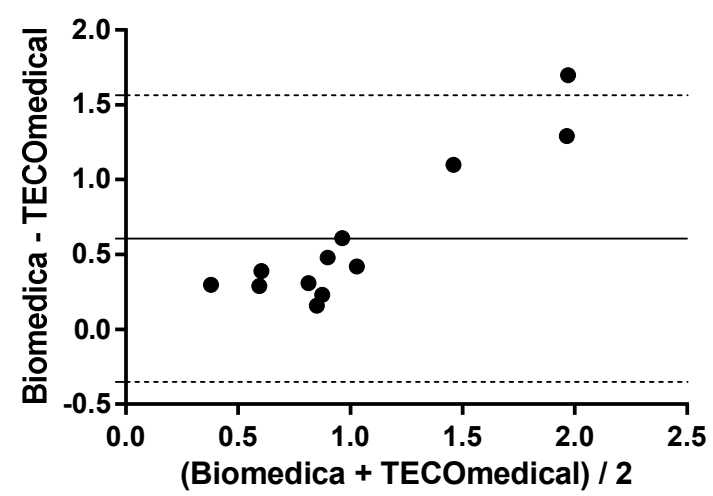

all sample types

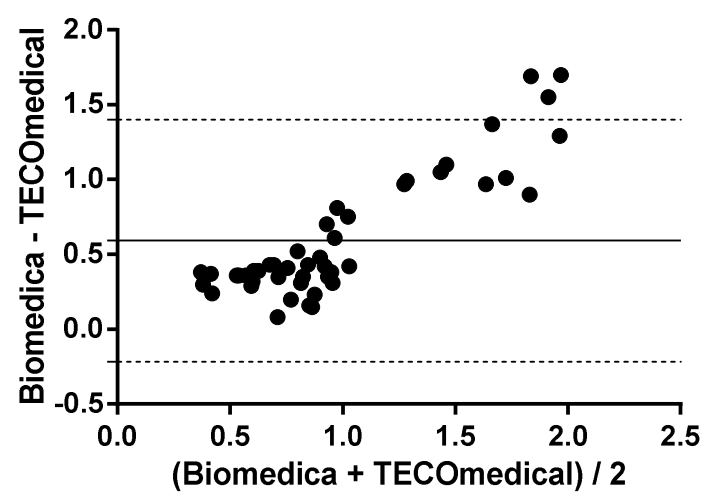

heparin

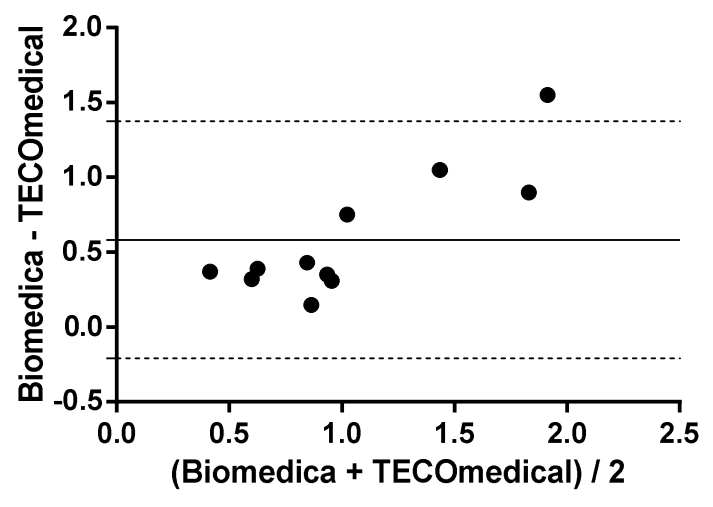

citrate

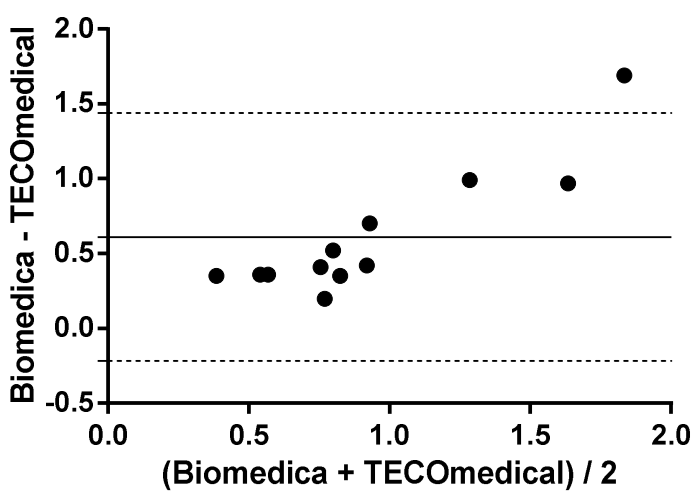

all sample types

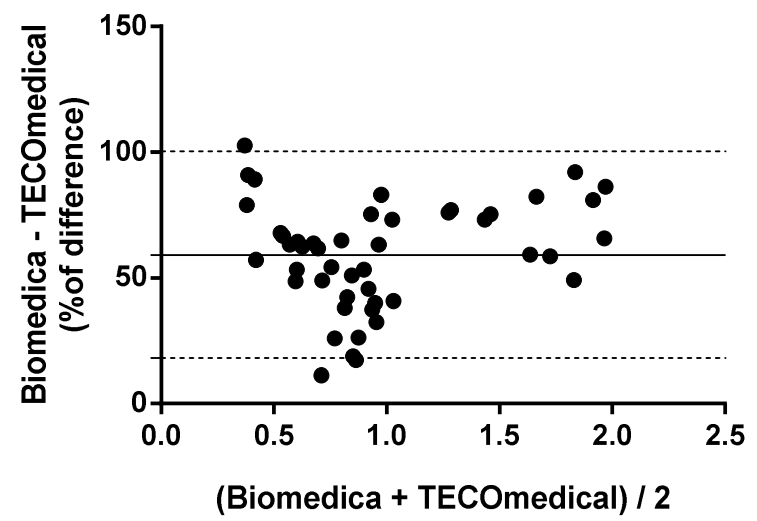

Figure 2. Bland-Altman difference plots visualizing the difference of the measurements obtained with the Biomedica and TECOmedical ELISAs. The differences or the bias (Biomedica - TECOmedical; Y-axis in $\mathrm{ng} / \mathrm{mL}$ ) is plotted versus the average of the two readings (Biomedica + TECOmedical / 2; X-axis in $\mathrm{ng} / \mathrm{mL}$ ). As an increase in variability of the differences is observed as the magnitude of the measurement increases, the lower right Bland-Altman plot displays the relationship between the average and the percentage of difference. Thin continuous horizontal lines represent the mean, thin dotted horizontal lines indicate the $95 \%$ limits of agreement (average difference $\pm 1.96 \mathrm{SD}$ of the difference). 

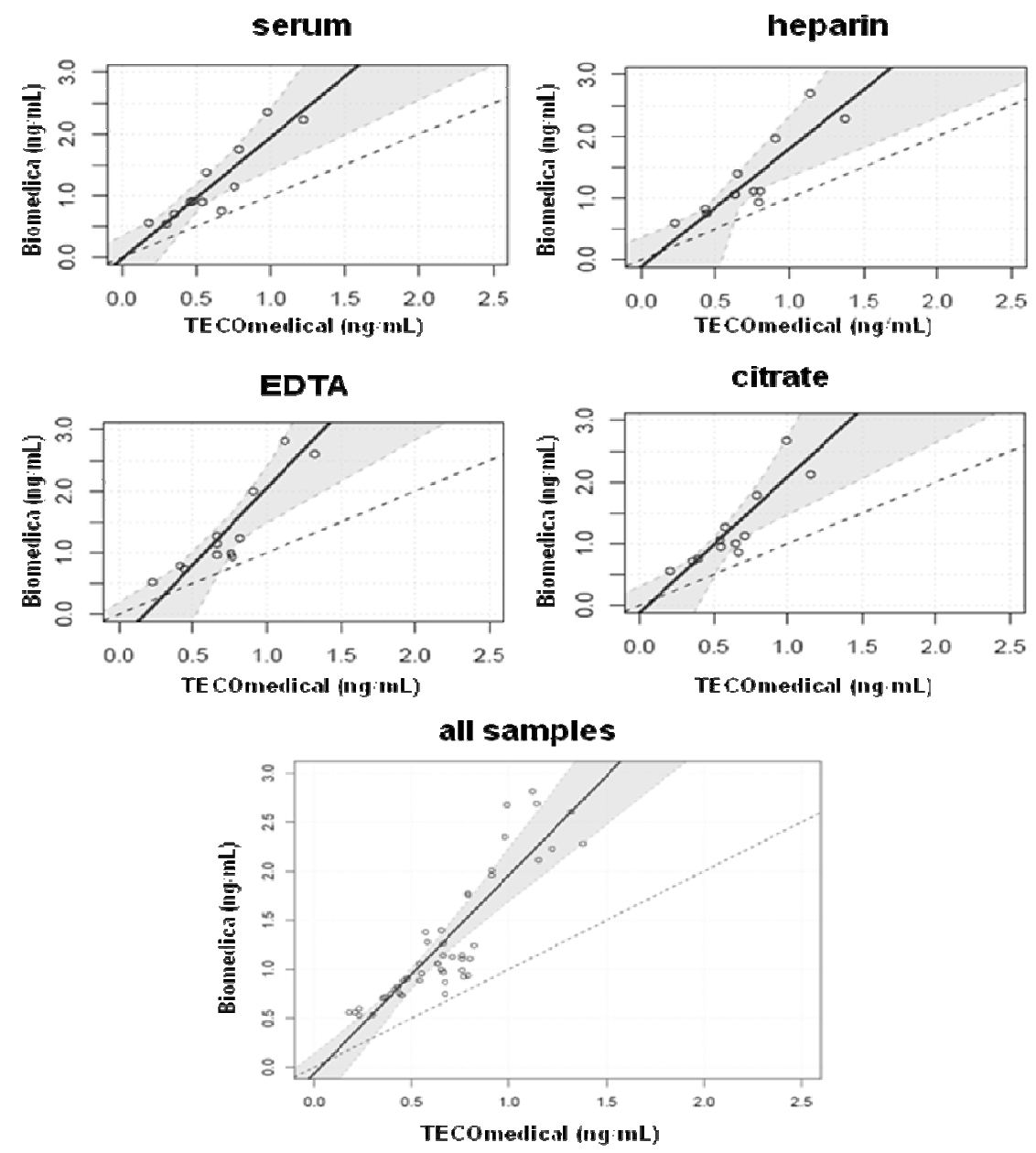

Figure 3. Plots displaying the Passing-Bablok regression analysis of the agreement between the Biomedica and TECOmedical ELISAs for various sample types as indicated. The solid line indicates the regression line, the black dotted lines show the line of identity and the gray dashed lines represent the $95 \%$ confidence interval for the regression line.

Table 5. Passing-Bablok regression analysis testing the agreement of the Biomedica and TECOmedical ELISAs and concordance correlation coefficients (CCC).

\begin{tabular}{|c|c|c|c|c|c|}
\hline $\begin{array}{l}\text { Biomedica vs. } \\
\text { TECOmedical }\end{array}$ & serum & heparin & EDTA & citrate & all samples \\
\hline slope & 1.969 & 1.905 & 2.504 & 2.203 & 2.024 \\
\hline $\begin{array}{l}95 \% \text { CI of } \\
\text { slope }\end{array}$ & $\begin{array}{c}1.136 \text { to } \\
3.105\end{array}$ & 0.962 to 3.196 & 1.351 to 4.167 & 1.171 to 4.500 & 1.571 to 2.632 \\
\hline intercept & -0.025 & 0.109 & -0.449 & -0.119 & -0.064 \\
\hline $\begin{array}{c}95 \% \text { CI of } \\
\text { intercept }\end{array}$ & $\begin{array}{c}-0.693 \text { to } \\
0.348\end{array}$ & $\begin{array}{c}-0.964 \text { to } \\
0.367\end{array}$ & -2.177 to 0.194 & -1.911 to 0.308 & -0.461 to 0.142 \\
\hline $\mathrm{CCC}$ & 0.40 & 0.40 & 0.38 & 0.33 & 0.39 \\
\hline $\begin{array}{c}95 \% \text { CI of } \\
\text { CCC }\end{array}$ & 0.15 to 0.60 & 0.14 to 0.61 & 0.15 to 0.58 & 0.11 to 0.53 & $\begin{array}{c}0.27 \text { to } \\
0.49\end{array}$ \\
\hline
\end{tabular}



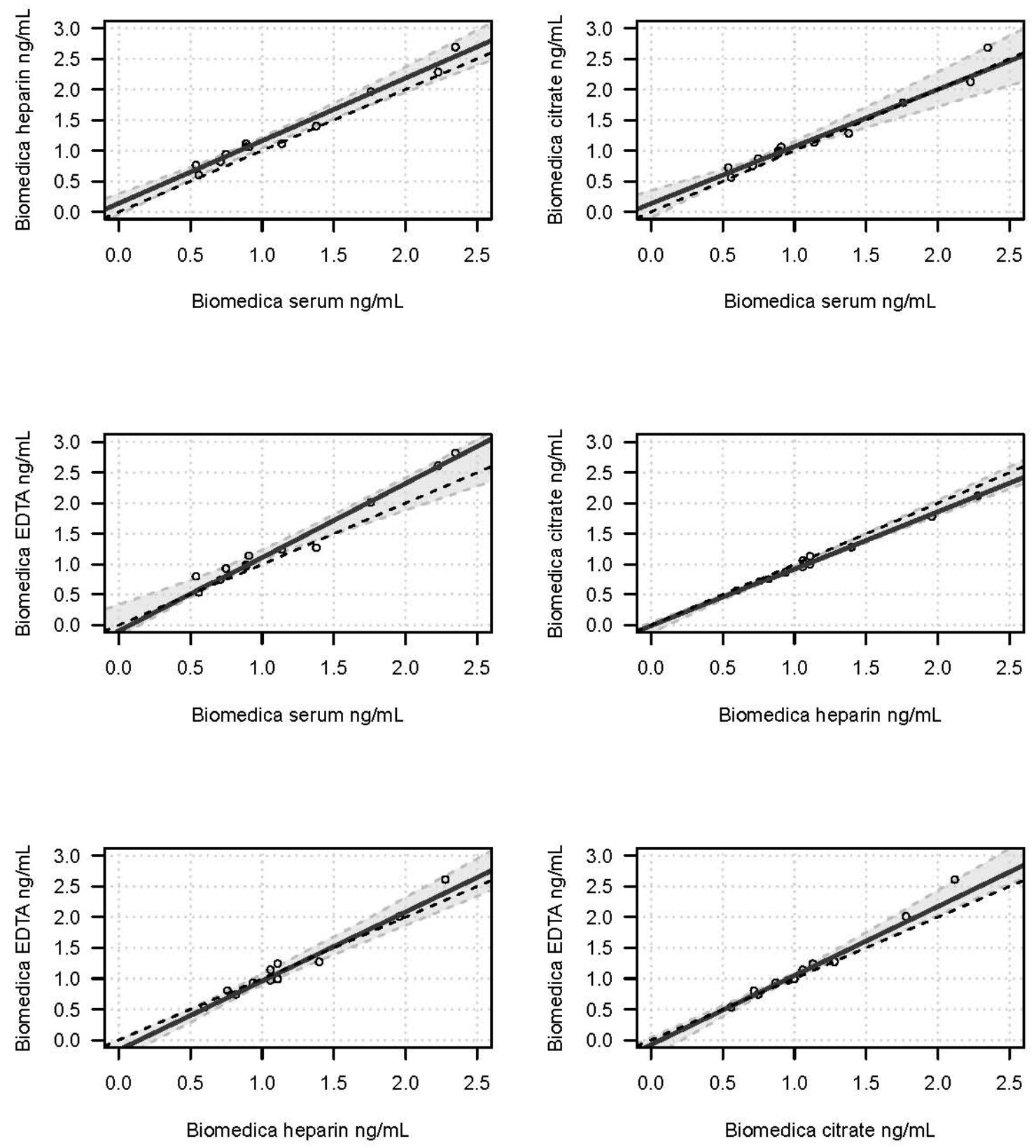

Figure 4A. Plots displaying the Passing-Bablok regression analysis of the agreement between the various samples types as indicated. Analysis was performed for results obtained by the Biomedica. The solid line indicates the regression line, the black dotted lines show the line of identity and the gray dashed lines represent the $95 \%$ confidence interval for the regression line. 

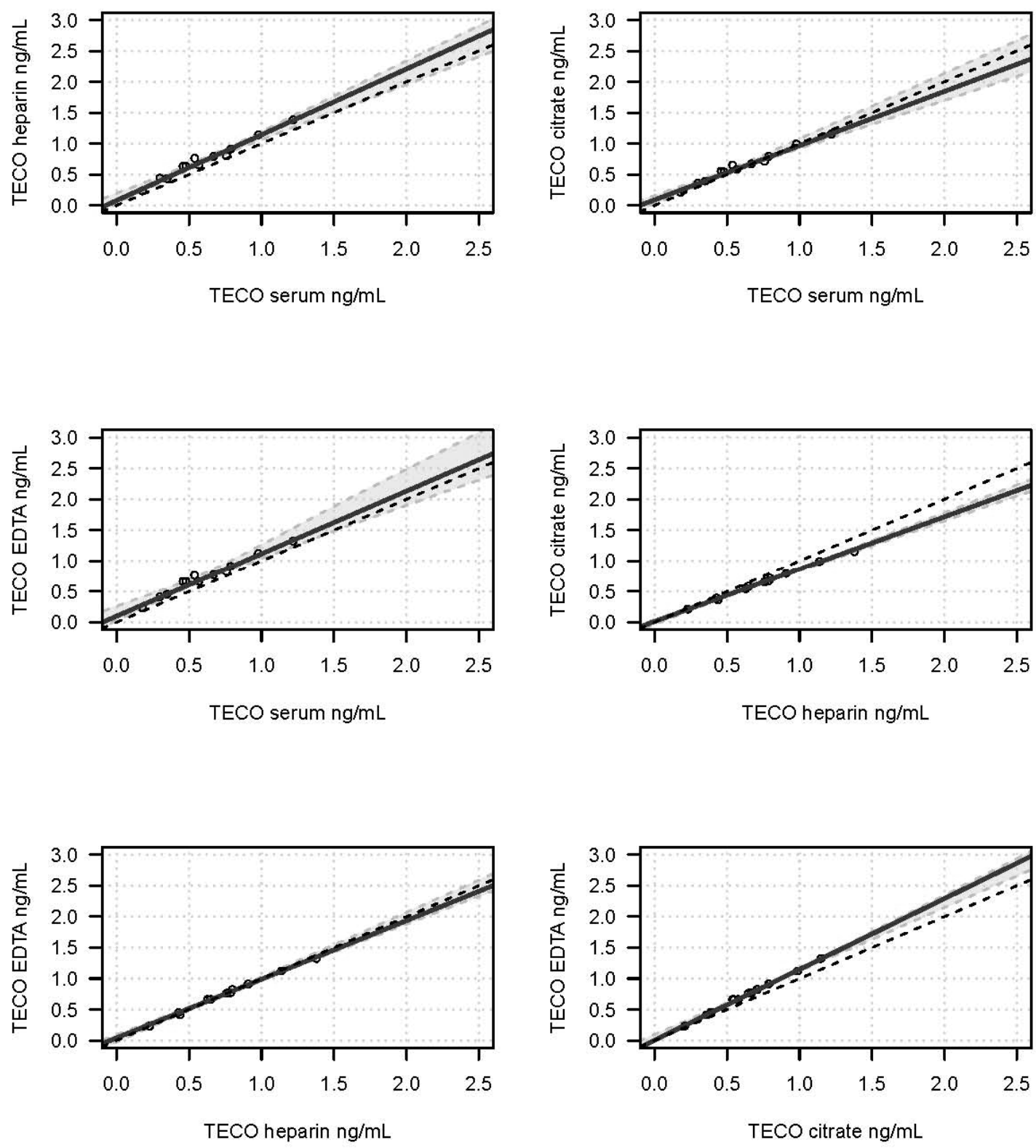

Figure 4B. Plots displaying the Passing-Bablok regression analysis of the agreement between the various samples types as indicated. Analysis was performed for results obtained by the TECOmedical ELISA. The solid line indicates the regression line, the black dotted lines show the line of identity and the gray dashed lines represent the $95 \%$ confidence interval for the regression line. 
Table 6. Passing-Bablok regression analysis testing the agreement of different sample types and concordance correlation coefficients (CCC).

\begin{tabular}{|c|c|c|c|c|c|c|}
\hline & $\begin{array}{l}\text { serum vs. } \\
\text { heparin }\end{array}$ & $\begin{array}{l}\text { serum vs. } \\
\text { EDTA }\end{array}$ & $\begin{array}{l}\text { serum vs. } \\
\text { citrate }\end{array}$ & $\begin{array}{c}\text { heparin vs. } \\
\text { EDTA }\end{array}$ & $\begin{array}{l}\text { heparin vs. } \\
\text { citrate }\end{array}$ & $\begin{array}{l}\text { citrate vs. } \\
\text { EDTA }\end{array}$ \\
\hline \multicolumn{7}{|l|}{ Biomedica } \\
\hline slope & 1.022 & 1.209 & 0.931 & 1.124 & 0.936 & 1.124 \\
\hline $95 \%$ CI of slope & $\begin{array}{c}0.857 \text { to } \\
1.200\end{array}$ & $\begin{array}{c}0.791 \text { to } \\
1.279\end{array}$ & $\begin{array}{c}0.686 \text { to } \\
1.184\end{array}$ & $\begin{array}{c}0.954 \text { to } \\
1.326\end{array}$ & $\begin{array}{c}0.879 \text { to } \\
1.087\end{array}$ & $\begin{array}{c}1.000 \text { to } \\
1.361\end{array}$ \\
\hline intercept & 0.140 & -0.096 & 0.136 & -0.163 & -0.011 & -0.076 \\
\hline $\begin{array}{l}95 \% \mathrm{CI} \text { of } \\
\text { intercept }\end{array}$ & $\begin{array}{c}-0.042 \text { to } \\
0.297\end{array}$ & $\begin{array}{c}-0.185 \text { to } \\
0.333\end{array}$ & $\begin{array}{c}-0.094 \text { to } \\
0.349\end{array}$ & $\begin{array}{c}-0.320 \text { to } \\
0.004\end{array}$ & $\begin{array}{c}-0.124 \text { to } \\
0.042\end{array}$ & $\begin{array}{c}-0.293 \text { to } \\
0.041\end{array}$ \\
\hline $\mathrm{CCC}$ & 0.96 & 0.94 & 0.97 & 0.98 & 0.99 & 0.97 \\
\hline $95 \%$ CI of CCC & $\begin{array}{c}0.89 \text { to } \\
0.99\end{array}$ & 0.85 to 0.98 & 0.92 to 0.99 & 0.95 to 0.99 & $\begin{array}{c}0.96 \text { to } \\
1.00\end{array}$ & 0.84 to 0.97 \\
\hline \multicolumn{7}{|l|}{ TECOmedical } \\
\hline slope & 1.064 & 1.016 & 0.877 & 0.947 & 0.851 & 1.144 \\
\hline $95 \%$ CI of slope & $\begin{array}{c}0.902 \text { to } \\
1.143\end{array}$ & $\begin{array}{c}0.842 \text { to } \\
1.213\end{array}$ & $\begin{array}{c}0.784 \text { to } \\
1.059\end{array}$ & $\begin{array}{c}0.892 \text { to } \\
1.042\end{array}$ & $\begin{array}{c}0.806 \text { to } \\
0.906\end{array}$ & $\begin{array}{c}1.022 \text { to } \\
1.180\end{array}$ \\
\hline intercept & 0.079 & 0.100 & 0.090 & 0.041 & 0.014 & 0.004 \\
\hline $\begin{array}{l}95 \% \mathrm{CI} \text { of } \\
\text { intercept }\end{array}$ & $\begin{array}{c}0.027 \text { to } \\
0.1964\end{array}$ & $\begin{array}{c}0.029 \text { to } \\
0.261\end{array}$ & $\begin{array}{c}0.025 \text { to } \\
0.161\end{array}$ & $\begin{array}{c}-0.024 \text { to } \\
0.092\end{array}$ & $\begin{array}{c}-0.032 \text { to } \\
0.043\end{array}$ & $\begin{array}{c}-0.016 \text { to } \\
0.097\end{array}$ \\
\hline $\mathrm{CCC}$ & 0.90 & 0.90 & 0.98 & 1.00 & 0.93 & 0.93 \\
\hline $95 \% \mathrm{CI}$ of $\mathrm{CCC}$ & $\begin{array}{c}0.77 \text { to } \\
0.96\end{array}$ & 0.76 to 0.96 & 0.94 to 0.99 & 0.99 to 1.00 & $\begin{array}{c}0.83 \text { to } \\
0.97\end{array}$ & 0.84 to 0.97 \\
\hline
\end{tabular}

Together with uncertainties in respect to the optimal pre-analytical handling, this inconsistency has limited the introduction of sclerostin measurement into general clinical practices. We therefore compared two commercially available sclerostin ELISAs in order to validate assay quality characteristics. Among the various commercially available sclerostin assays, the ELISAs by TECOmedical and Biomedica are currently the most widespread applied assays. Moreover, we particularly evaluated the influence of preanalytical variables including storage time and blood material.

We performed the study on purpose in HD patients since they represent a cohort of particular interest for sclerostin assessment. In summary, while storage of unprocessed samples for up to 4 hours at room temperature has only minor influence on measured sclerostin levels, this study clearly reveals a disagreement and strong proportional error between the Biomedica and TECOmedical ELISAs and identifies a bias among different blood sample types.plasma samples.

Our results confirm the fulfillment of the stability criteria suggested by the European Medicines Agency for both assays and demonstrated the capability of the sample material to retain the initial property of the measured constituent at room temperature within the tested time frame, especially when stored as plasma samples ${ }^{19}$. Thus we can conclude that routine blood sample handling in dialysis units seems to be appropriate for sclerostin assessment as our data indicate that it is feasible to store samples from HD patients for up to 4 hours before centrifugation and further analysis. In order to be acceptable for widespread clinical use in ESRD patients, it is necessary to ensure that sample dilution and matrix effects have no adverse effects on the integrity of the results obtained. In this respect, the TECOmedical assay yielded a good accuracy with an adequate dilution linearity/parallelism and a satisfactory spike recovery in the tested samples 
- PROBLEM: disagreement between commercially avaflable assays and uncertainty in respect to the proper pre-analytical handling of blood samples

- RESULTS: validation of the two most common and commercially avallable sclerostin ELISAs revealed (1) a poor agreement of the two assays with a strong proportional but no systematic bias and (2) a systematic but not proportional bias between serum and plasma samples with higher values for plasma samples.

- NEED: to establish standardized guidelines for analytical methods.

from patients with ESRD. The Biomedica assay showed lower accuracy with an over-recovery in the dilution series and an under-recovery in the spike testing. This result points towards differences in sample-matrix-interferences between the two assays.

The present study shows that among patients with ESRD undergoing hemodialysis, results derived from the two sclerostin ELISAs should not be directly compared due to the poor agreement with a relevant proportional bias. Prior studies already investigated the Biomedica and TECOmedical ELISAs for detection of sclerostin in various patient populations. In accordance with our results, McNulty et al. identified both in serum and heparin plasma samples obtained from healthy, middle-aged individuals considerably higher sclerostin levels with the Biomedica assay (1,4-fold in serum, 1,8-fold in heparin plasma samples) ${ }^{13}$. Measured sclerostin levels were found to be lower in serum than in plasma samples. Similar, Durosier et al. analyzed sclerostin levels in serum of healthy elderly individuals and measured 2-fold higher values with the Biomedica assay compared to the TECOmedical ELISA ${ }^{14}$. A relevant limitation of the interpretation of these studies is that the authors applied only a correlation analysis determining linear association instead of the more adequate Passing-Bablok regression analysis testing the true agreement of the assays ${ }^{20}$. The non-parametric regression analysis developed by Passing and Bablok allows estimation of analytical methods agreement and possible systematic and/or proportional bias between them and overcomes the assumptions of the classical linear regression ${ }^{21}$. Our study therefore substantially extends previous validation studies of the Biomedica and TECOmedical ELISAs. Costa et al. recently identified in hospitalized patients or patients with metabolic bone diseases and CKD a strong correlation between sclerostin levels detected by the two ELISAs and reported that the mean concentration of sclerostin in serum samples differed by only $2.9 \%$ due to a "slight systematic and proportional" error ${ }^{15}$. The reason for this inconsistent magnitude of differences among the studies remains unclear ${ }^{16}$. Matrix interference effects might be variably present or dominant in analyzed samples from various patient populations. Furthermore, the presence of circulating sclerostin in a dimeric form or sclerostin fragments, as well as the formation of protein complexes might differentially affect the recognition by antibodies used in the assays ${ }^{16,22,23}$.

Although serum and plasma specimens are considered equivalent for many assays ${ }^{24}$, previous studies and our results documented that sclerostin values measured in serum were generally lower than in plasma ${ }^{13,14,25}$. Further exploring this observation, Passing-Bablok analysis of the TECOmedical assay (but not Biomedica assay) now provided evidence for a systematic bias between serum and each of the plasma samples. It remains speculative whether the addition of anticoagulants like EDTA, citrate and heparin influences the accessibility of recognizable epitopes and thus affects the values of measured sclerostin. Our data do not allow concluding whether serum or plasma yields the "true" level of circulating sclerostin. For the first time regarding sclerostin measurement, we provide a systematic comparison of 3 different plasma types, namely heparin, EDTA and citrate plasma. Regression analysis of results obtained from the TECOmedical assay demonstrated a proportional error between citrate and EDTA or heparin plasma with lower sclerostin values 
measured in citrate plasma. Independent of the assay applied, our study reveals the absence of a systematic error between all plasma sample types.

In summary, our data indicate that sclerostin measurements in HD patients are reliable in both serum and plasma. However, serum and plasma sclerostin levels are not directly comparable. Due to the better stability during storage, we recommend using plasma samples for analysis of sclerostin in patients with ESRD. Results obtained by the two ELISAs should not be used interchangeably. In conclusion, clear annotation of the applied sclerostin ELISA type, as well as blood sample type, is mandatory for interpretation of sclerostin results in HD patients. With the validation of the two most commonly used sclerostin immunoassays, our data allows in the context of ESRD a more differentiated judgement of reported circulating sclerostin levels among various studies and contributes to the development and final establishment of sclerostin as a reliable and clinical relevant biomarker, although assay validation studies in larger populations will be necessary for further confirmation.

\section{Acknowledgments}

The authors gratefully thank Dr. Andras Keszei, Department of Medical Informatics, University Hospital of the RWTH Aachen, Germany, for advices and assistance in statistical analysis. The authors gratefully acknowledge grant support from the NIH (R01HL081863 to WAB).

\section{Conflict of Interest:}

M.H. is an employee of TECOmedical. The other authors declare to have no conflict of interest with respect to the present manuscript.

\section{References:}

1. Moe S, Drueke T, Cunningham J, et al. Definition, evaluation, and classification of renal osteodystrophy: a position statement from Kidney Disease: Improving Global Outcomes (KDIGO). Kidney Int. 2006;69:19451953.

2. Winkler DG, Sutherland MK, Geoghegan JC, et al. Osteocyte control of bone formation via sclerostin, a novel BMP antagonist. EMBO J. 2003;22:6267-6276.

3. Brandenburg VM., D'Haese P, Deck A, et al. From skeletal to cardiovascular disease in 12 steps-the evolution of sclerostin as a major player in CKD-MBD. Pediatr Nephrol. 2016;31:195-206

4. Sabbagh Y, Graciolli FG, O'Brien S, et al. Repression of osteocyte Wnt/beta-catenin signaling is an early event in the progression of renal osteodystrophy. $J$ Bone Miner Res. 2012;27:1757-1772.

5. Ishimura E, Okuno S, Ichii M, et al. Relationship between serum sclerostin, bone metabolism markers, and bone mineral density in maintenance hemodialysis patients. J Clin Endocrinol Metab. 2014;99:4315-4320.

6. Drechsler C, Evenepoel P, Vervloet MG, et al. High levels of circulating sclerostin are associated with better cardiovascular survival in incident dialysis patients: results from the NECOSAD study. Nephrol Dial Transplant. 2015;30:288-293.

7. Goncalves FL, Elias RM, dos Reis LM, et al. Serum sclerostin is an independent predictor of mortality in hemodialysis patients. BMC Nephrol. 2014; 15:190.

8. Claes KJ, Viaene L, Heye S, et al. Sclerostin: Another vascular calcification inhibitor? J Clin Endocrinol Metab. 2013;98:3221-3228.

9. Brandenburg VM, Kramann R, Koos R, et al. Relationship between sclerostin and cardiovascular calcification in hemodialysis patients: a cross-sectional study. BMC Nephrol. 2013;14:219.

10. Pelletier S, Confavreux CB, Haesebaert J, et al. Serum sclerostin: the missing link in the bone-vessel cross-talk in hemodialysis patients? Osteoporos Int. 2015;26:2165-2174

11. Chau CH, Rixe O, McLeod H, Figg WD. Validation of analytic methods for biomarkers used in drug development. Clin Cancer Res. 2008;14:5967-5976.

12. Cantor T, Yang Z, Caraiani N, Ilamathi E. Lack of comparability of intact parathyroid hormone measurements among commercial assays for end-stage renal disease patients: implication for treatment decisions. Clin Chem. 2006;52:1771-1776.

13. McNulty M, Singh RJ, Li X, Bergstralh EJ, Kumar R. Determination of serum and plasma sclerostin concentrations by enzyme-linked immunoassays. J Clin Endocrinol Metab 2011;96:E1159-1162.

14. Durosier C, van Lierop A, Ferrari S, et al. Association of circulating sclerostin with bone mineral mass, microstructure, and turnover biochemical markers in healthy elderly men and women. J Clin Endocrinol Metab. 2013;98:3873-3883.

15. Costa AG, Cremers S, Dworakowski E, LazarettiCastro M, Bilezikian JP. Comparison of two commercially available ELISAs for circulating sclerostin. Osteoporos Int. 2014;25:1547-1554.

16. Clarke BL, Drake MT. Clinical utility of serum sclerostin measurements. Bonekey Rep. 2013;2:361.

17. Bland JM, Altman DG. Statistical methods for assessing agreement between two methods of clinical measurement. Lancet. 1986;1:307-310.

18. Moysés RM, Jamal SA, Graciolli FG, et al. Can we compare serum sclerostin results obtained with different assays in hemodialysis patients?: Int Urol Nephrol. 2015;47:847-50.

19. Boterman M, Doig $\mathrm{M}$, Breda $\mathrm{M}$, et al. Recommendations on the interpretation of the new 
European Medicines Agency Guideline on Bioanalytical Method Validation by Global CRO Council for Bioanalysis (GCC). Bioanalysis. 2012;4:651-660.

20. Zaki R, Bulgiba A, Ismail R, Ismail NA. Statistical methods used to test for agreement of medical instruments measuring continuous variables in method comparison studies: a systematic review. PLoS One. 2012;7:e37908.

21. Passing H, Bablok. A new biometrical procedure for testing the equality of measurements from two different analytical methods. Application of linear regression procedures for method comparison studies in clinical chemistry, Part I. J Clin Chem Clin Biochem. 1983;21:709-720.

22.Veverka V, Henry AJ, Slocombe PM, et al. Characterization of the structural features and interactions of sclerostin: molecular insight into a key regulator of Wnt-mediated bone formation. J Biol Chem. 2009;284:10890-10900.
23. Hernandez P, Whitty C, John Wardale R, Henson FM. New insights into the location and form of sclerostin. Biochem Biophys Res Commun. 2014;446:1108-1113.

24. Miles RR, Roberts RF, Putnam AR, Roberts WL. Comparison of serum and heparinized plasma samples for measurement of chemistry analytes. Clin Chem. 2004;50:1704-1706.

25. van Lierop $\mathrm{AH}$, van der Eerden $\mathrm{AW}$, et al. Circulating sclerostin levels are decreased in patients with endogenous hypercortisolism and increase after treatment. J Clin Endocrinol Metab. 2012;97:E19531957.

DISCOVERIES is a peer-reviewed, open access, online, multidisciplinary and integrative journal, publishing high impact and innovative manuscripts from all areas related to MEDICINE, BIOLOGY and CHEMISTRY; @ 2016, Applied Systems 